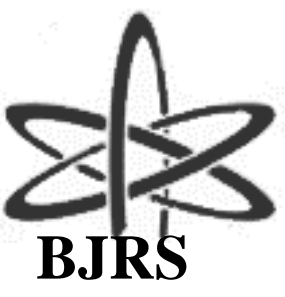

\author{
BRAZILIAN JOURNAL \\ $\mathrm{OF}$ \\ RADIATION SCIENCES \\ 05-03-A (2017) 01-09
}

\title{
Desenvolvimento de técnicas de bioanálise in vitro para monitoração individual de ${ }^{32} \mathrm{P}$
}

\author{
A.P. F. Almeida; A.L.A. Dantas; W.O.Sousa, B.M. Dantas \\ Instituto de Radioproteção e Dosimetria /Divisão de Dosimetria \\ Av. Salvador Allendes/n, CEP 22783-127 \\ Rio de Janeiro-RJ, Brasil \\ bmdantas@ird.gov.br
}

\section{RESUMO}

$\mathrm{O}{ }^{32} \mathrm{P}$ é usualmente manipulado na forma de fontes líquidas não seladas em instalações médicas, instituições de pesquisa e ensino, e na produção industrial de radiofármacos. Tais práticas representam um risco de exposição interna em tarefas de rotina e em casos de acidentes. A estimativa da incorporação de ${ }^{32} \mathrm{P}$ pode ser realizada por meio de técnicas de bioanálise in vitro. Este artigo apresenta a metodologia de análise de ${ }^{32} \mathrm{P}$ em amostras biológicas, aplicável à monitoração individual interna utilizando técnicas de cintilação líquida. Baseado nos resultados experimentais pode-se concluir que a técnica apresenta sensibilidade suficiente para avaliar incorporações acidentais de atividades que resultem em doses efetivas comprometidas da ordem de $1 \mathrm{mSv}$

Palavras-chave: Bioanálise, fósforo-32, Quenching colirimétrico,Efeito Cerenkov

\begin{abstract}
${ }^{32} \mathrm{P}$ is usually handled in the form of liquid unsealed sources in medical facilities, in research and teaching institutions, and in industrial radiopharmaceutical production plants. Such practices represent a risk of internal exposure in routine activities and in case of accidents. The estimation of ${ }^{32} \mathrm{P}$ incorporation can be accomplished through in vitro bioassay. This paper presents a methodology to analyze ${ }^{32} \mathrm{P}$ in biological samples, applicable to internal individual monitoring using liquid scintillation technique. Based on the experimental results it is possible to conclude that the technique presents enough sensitivity to detect activities that result in committed effective dose in the order of $1 \mathrm{mSv}$.
\end{abstract}

Keywords: Bioassay, phosphorus-32, Colorimetric Quenching, Cerenkov Effect. 


\section{INTRODUÇÃO}

No Brasil, um número significativo de indivíduos está exposto a radionuclídeos emissores beta, por exemplo ${ }^{32} \mathrm{P},{ }^{3} \mathrm{H},{ }^{14} \mathrm{C},{ }^{89} \mathrm{Sr}$ e ${ }^{90} \mathrm{Sr}$, na produção e utilização de radiofármacos para fins médicos, e também em instituições de ensino e pesquisa biomédica. A manipulação rotineira destes radionuclídeos envolve riscos à saúde devido à possibilidade de ocorrência de incorporações acidentais e consequente exposição interna [1].

De acordo com a norma básica de Comissão Nacional de Energia Nuclear [2] a exposição de indivíduos deve ser restringida de tal modo que a dose efetiva decorrente de exposições à radiação não exceda o limite anual de $1 \mathrm{mSv}$ para público e $20 \mathrm{mSv}$ em uma média de 5 anos para trabalhadores. Assim, os indivíduos potencialmente expostos devem ser submetidos à monitoração através de técnicas apropriadas.

A monitoração da incorporação de ${ }^{32} \mathrm{P}$ inclui a aplicação de técnicas de bioanálise assim como a interpretação dos resultados obtidos visando o cálculo da dose interna. O modelo biocinético do radionuclídeo, descrito pela Comissão Internacional de Proteção Radiológica (ICRP), na sua publicação 30 [3], foi estudado com relação à sua distribuição no corpo humano e excreção através da urina, após incorporação pelas vias mais prováveis em situações de exposição ocupacionais. Os compostos de ${ }^{32} \mathrm{P}$, após atingirem a corrente sanguínea depositam-se no tecido ósseo, sendo também excretados via urina, podendo ser determinados através de técnicas de bioanálise in vitro [4].

$\mathrm{O}{ }^{32} \mathrm{P}$ pode ser determinado utilizando a técnica de cintilação líquida com ou sem o uso de solução cintiladora. A técnica de cintilação líquida sem o uso de solução cintiladora é conhecida como Efeito Cerenkov sendo a mais indicada para detecção do ${ }^{32} \mathrm{P}$, pois evita a interferência de radionuclídeos emissores de partículas beta de baixa energia $\left({ }^{3} \mathrm{H},{ }^{14} \mathrm{C},{ }^{33} \mathrm{P},{ }^{35} \mathrm{~S},{ }^{45} \mathrm{Ca}\right)$, além de possuir baixa eficiência para os emissores de raios gama $\left({ }^{22} \mathrm{Na},{ }^{51} \mathrm{Cr},{ }^{54} \mathrm{Mn},{ }^{59} \mathrm{Fe},{ }^{60} \mathrm{Co},{ }^{65} \mathrm{Zn},{ }^{75} \mathrm{Se}\right)$.

Neste estudo, o termo calibração foi aplicado de acordo com a definição apresentada no documento VIM [5], onde calibração é definida como uma operação que estabelece, sob condições especificadas, uma relação entre os valores de medição fornecidos por padrões e as indicações correspondentes geradas pelo equipamento utilizado. 


\section{MATERIAIS E MÉTODOS}

Foi desenvolvida no laboratório de bioanálise in vitro a metodologia de calibração do cintilador líquido modelo Quantulus 1220 (Perkin Elmer) para determinação de ${ }^{32} \mathrm{P}$ em amostras biológicas, tendo sido utilizada uma solução de $10 \mathrm{mg} \cdot \mathrm{g}^{-1}$ de $\mathrm{Na}_{2} \mathrm{H}_{32} \mathrm{PO}_{4}$ em $\mathrm{HCl} 0,1 \mathrm{M}$, contendo padrão de ${ }^{32} \mathrm{P}$ com atividade de 47,2 kBq/g ${ }^{-1}$. Dois tipos de calibração foram realizados, um utilizando solução cintiladora e outro sem a de solução cintiladora.

A sensibilidade da técnica foi avaliada com base na determinação da atividade mínima detectável (AMD). A estimativa de incorporação mínima detectável (IMD) e sua comparação com a dose efetiva mínima detectável (DEMD) foi determinada utilizando os modelos biocinéticos e dosimétricos desenvolvidos pela ICRP, na sua publicação 78 [6]. A ICRP classifica os compostos em três categorias, de acordo com a solubilidade no sistema respiratório: Tipo F (muito solúvel), Tipo M (moderadamente solúvel) e Tipo S (insolúvel).

\subsection{Calibração do Cintilador Líquido - Quantulus 1220 (PERKIN ELMER)}

\subsubsection{Calibração com Solução cintiladora}

Três amostras padrões foram preparadas para calibração do cintilador, usando a fonte certificada de ${ }^{32} \mathrm{P}$ (CT/120053/12/0264 - LEA - Laboratorie Etalons d'Activité). Foram adicionadas aos frascos uma atividade conhecida de ${ }^{32} \mathrm{P}$ em $19 \mathrm{~mL}$ de solução cintiladora (Optiphase-Hi-Safe3) e $1 \mathrm{~mL}$ de $\mathrm{H}_{2} \mathrm{O}$. Os frascos foram contados no cintilador durante 100 min para determinação da eficiência de detecção. Posteriormente foram adicionadas gotas de $\mathrm{Na}_{2} \mathrm{CrO}_{4}$ nos frascos, com a finalidade de provocar um quenching colorimétrico.

\subsubsection{Calibração sem solução cintiladora - Efeito Cerenkov}

Foram preparadas 3 amostras padrões para calibração, usando uma fonte certificada de ${ }^{32} \mathrm{P}$. Foi adicionada aos frascos atividade conhecida de ${ }^{32} \mathrm{P}$ em $10 \mathrm{~mL}$ de $\mathrm{H}_{2} \mathrm{O}$. Os frascos foram levados ao cintilador e contados durante 100 min para determinação da eficiência de contagem do Cintilador líquido - Quantulus 1220 (PERKIN ELMER). Numa segunda etapa foram adicionados mais $10 \mathrm{~mL}$ 
de água em 2 dos frascos preparados anteriormente, com o objetivo de verificar se o aumento de volume provocaria alteração na eficiência de deteção.

\subsection{Determinação da dose Mínima Detectável (IMD) e a Dose Efetiva Mínima Detectável (DEMD)}

A Incorporação Mínima Detectável (IMD) e a Dose Efetiva Mínima Detectável (DEMD) podem ser calculadas através das equações 1 e 2 respectivamente:

$$
\mathbf{I M D}=\mathbf{A M D} / \mathbf{m}(\mathbf{t})
$$

Onde:

IMD - Incorporação mínima detectável (Bq);

AMD - Atividade mínima detectável da técnica $(\mathrm{Bq})$;

$\mathrm{m}(\mathrm{t})$ - Valor da fração de excreção de atividade, em $\mathrm{Bq} / \mathrm{Bq}$, no compartimento do modelo biocinético (urina), em função do tempo (t) decorrido após a incorporação do radionuclídeo de interesse, para o cenário de incorporação avaliado.

$$
\text { DEMD = IMD . e (50) }
$$

Onde:

DEMD - Dose efetiva mínima detectável (Sv);

IMD - Incorporação mínima detectável (Bq);

e(50) - Coeficiente de conversão de dose para o cenário de incorporação avaliado (Sv/Bq).

Para a determinação das IMD e DEMD, foram utilizadas as frações de excreção e coeficientes de conversão de dose para o cenário de ingestão, de composto tipo $\mathrm{M}$, utilizando o software AIDE versão 6.0 [7]. 


\section{RESULTADOS E DISCUSSÃO}

\subsection{Calibração do Cintilador Líquido - Quantulus 1220 (PERKIN ELMER)}

\subsubsection{Calibração com Solução cintiladora}

A eficiência média de contagem, para o cintilador líquido, encontrada na análise dos 3 padrões preparados, foi de $100 \%$.

A avaliação da incorporação de ${ }^{32} \mathrm{P}$ em uma situação rotina e/ou acidente é feita através da coleta de amostras de urina. Como diferentes amostras de urinas apresentam diferentes concentrações e coloração, é necessário avaliar se a eficiência do cintilador líquido varia de forma significativa em função da interferência (quenching) provocada pela concentração ou cor da amostra de urina. A adição da solução de $\mathrm{Na}_{2} \mathrm{CrO}_{4}$ objetiva provocar uma variação no nível de quenching da amostra, em função do chamado quenching colorimétrico.

Os resultados encontrados após a adição de $\mathrm{Na}_{2} \mathrm{CrO}_{4}$ (Tabela 1) indicam que não houve uma variação significativa na eficiência de contagem, mesmo com uma variação significativa no nível de quenching da amostra. A eficiência média encontrada permaneceu em torno de $100 \%$ com uma pequena variação do nível de quenching chegando a uma eficiência média de $98 \%$ com uma variação significativa no nível de quenching.

Tabela 1: Efeito da adição do $\mathrm{Na}_{2} \mathrm{CrO}_{4}$ sobre a eficiência de deteção.

\begin{tabular}{cccc}
\hline Amostra & $\mathrm{Na}_{2} \mathrm{CrO}$ 4 & Eficiência & Média \\
\hline 1 & $\mathrm{~N}$ & 0,9991 & \\
2 & $\mathrm{~N}$ & 0,9781 & \\
3 & $\mathrm{~N}$ & 1,0719 & $\mathbf{1 , 0 1 6 3}$ \\
4 & $\mathrm{~S}$ & 1,0128 & \\
5 & $\mathrm{~S}$ & 1,0080 & \\
6 & $\mathrm{~S}$ & 1,0123 & $\mathbf{1 , 0 1 1 0}$ \\
\hline $\mathrm{N}=$ sem adição de $\mathrm{Na}_{2} \mathrm{CrO}_{4}$ & $\mathrm{~S}=$ com adição de $\mathrm{Na}_{2} \mathrm{CrO}_{4}$
\end{tabular}




\subsubsection{Calibração do cintilador sem solução cintiladora - Efeito Cerenkov}

A eficiência média de contagem encontrada considerando o volume inicial de $\mathrm{H}_{2} \mathrm{O}$ de $9 \mathrm{~mL}$ foi 49,9\%. Com a adição de mais $10 \mathrm{~mL}$ de $\mathrm{H}_{2} \mathrm{O}$ a eficiência média encontrada foi de 48,9\%. Os resultados das duas calibrações indicam que não houve uma variação significativa na eficiência de contagem em função do aumento do volume, indicando que a eficiência de contagem parece independer do volume de água presente.

\subsection{Incorporação Mínima Detectável (IMD) e Dose Efetiva Mínima Detectável (DEMD).}

As Figuras 1 e 2 apresentam as estimativas de incorporações mínimas detectáveis (IMD) e doses efetivas mínimas detectáveis (DEMD) respectivamente, tendo sido calculadas a partir da incorporação de ${ }^{32} \mathrm{P}$ ao nível da atividade mínima detectável (AMD) da técnica, cujo valor foi de 100 Bq. Esta estimativa considera as Frações de retenção, m(t), de atividade acumulada no compartimento urina em 24 horas, em função do tempo decorrido após a incorporação. A dose efetiva comprometida ( $\left.\mathrm{e}_{50}\right)$, para o cenário avaliado é de valor $2,39 \times 10^{-6} \mathrm{mSv}$.

Figura 1: Variação da IMD em função do tempo após incorporação única via ingestão

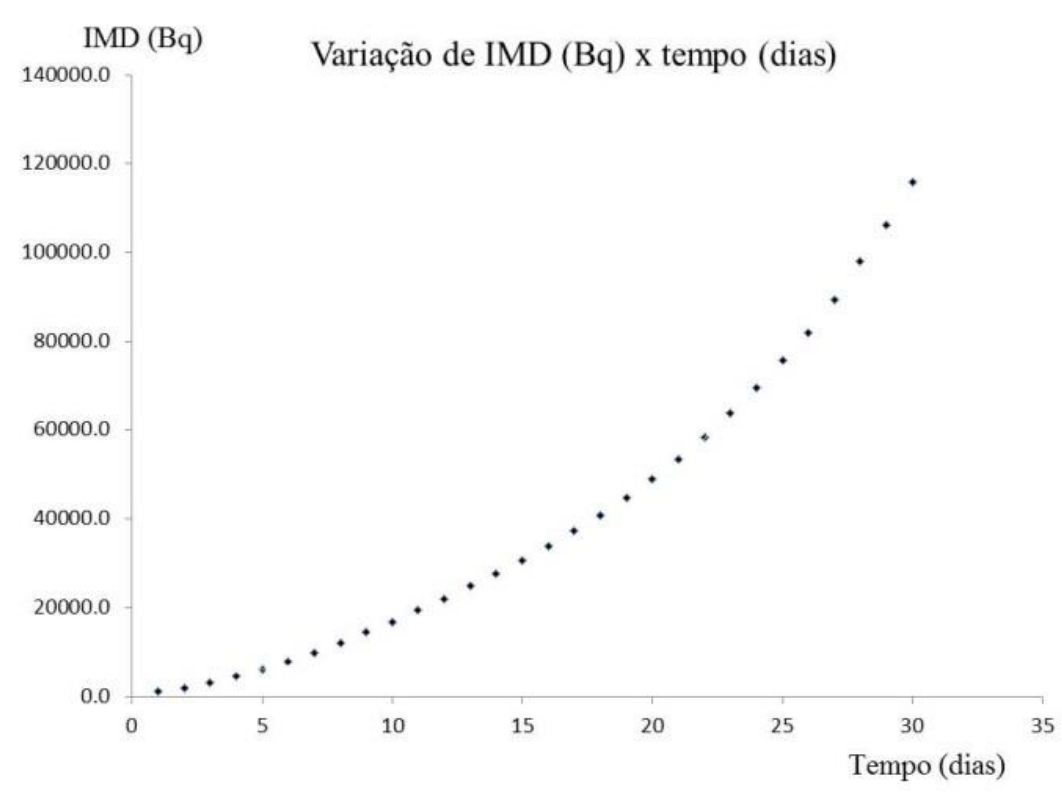


Figura 2: Variação da DEMD em função do tempo após incorporação única via ingestão



A figura 3 apresenta a variação da atividade de ${ }^{32} \mathrm{P}(\mathrm{Bq})$ em amostra de urina de 24 horas, supondose a incorporação única, via ingestão de 418.410 Bq, atividade que resulta em dose efetiva comprometida de $1 \mathrm{mSv}$.

Figura 3: Modelagem de excreção de ${ }^{32} \mathrm{P}$ em amostras de urina de 24 horas, após incorporação de atividade que resulte em dose efetiva comprometida de $1 \mathrm{mSv}$.

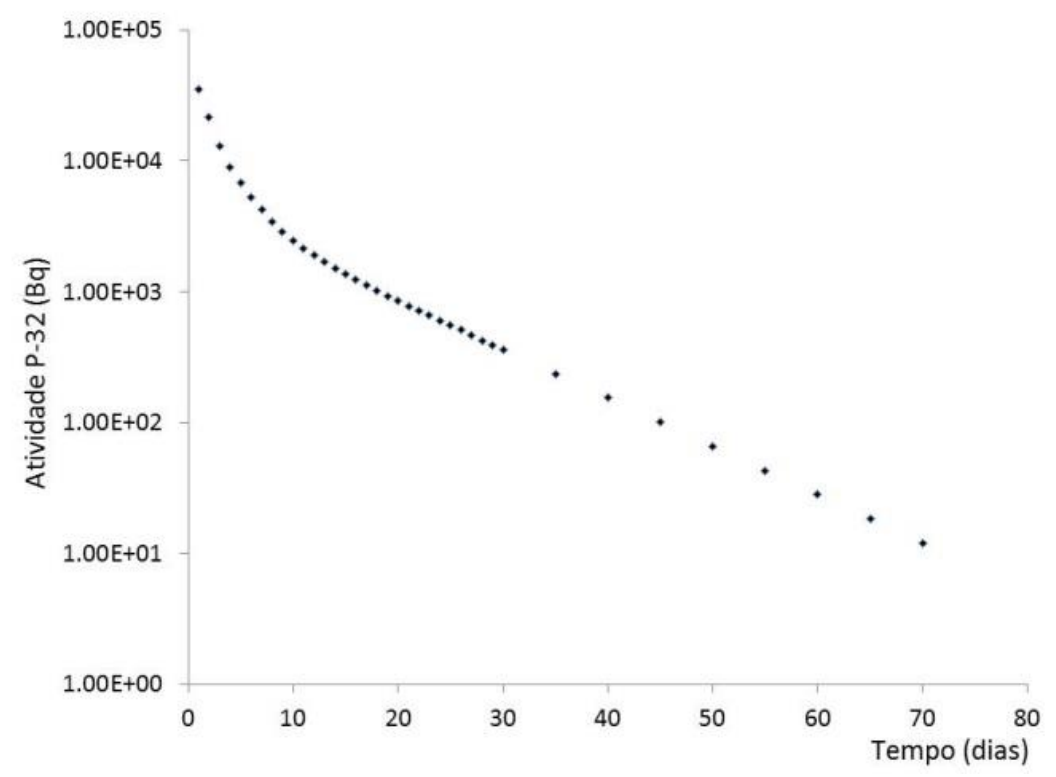


Verifica-se que a técnica, que apresenta atividade mínima detetável de $100 \mathrm{~Bq}$, é aplicável para medição de ${ }^{32} \mathrm{P}$ até 45 dias após a incorporação do radionuclídeo, via ingestão. Isto significa que até 45 dias é possível detectar ${ }^{32} \mathrm{P}$ em urina, decorrente da incorporação de uma atividade que resulte em dose efetiva comprometida da ordem de $1 \mathrm{mSv}$.

\section{CONCLUSÃO}

A utilização de solução cintiladora permitiu alcançar uma eficiência superior a $100 \%$ em relação ao observado pelo efeito Cerenkov, que apresentou eficiência de $50 \%$.

De acordo com os resultados das simulações realizadas com o programa AIDE, conclui-se que a técnica apresentou sensibilidade suficiente para detecção de ${ }^{32} \mathrm{P}$ em amostras biológicas, podendo ser utilizada em monitoração in vitro de incorporações acidentais únicas de atividades que resultem em doses efetivas comprometidas da ordem de $1 \mathrm{mSv}$.

\section{AGRADECIMENTOS}

Os autores agradecem ao apoio financeiro do CNPq relativo à bolsa da aluna A.P.F. Almeida, o que possibilitou a realização deste estudo.

\section{REFERÊNCIAS}

[1] IAEA - International Atomic Energy Agency. Assessment of Occupational Exposure Due to Intakes of Radionuclides Safety Standards Series RS-G-1.2, 1999

[2] CNEN - Comissão Nacional de Energia Nuclear. Diretrizes Básicas de Proteção Radiológica. Norma CNEN-NN-3.01, 2014

[3] ICRP - International Commission on Radiological Protection. Limits for Intakes of Radionuclides by Workers. Publication 30, 1980 
[4] IAEA - International Atomic Energy Agency. Methods for Assessing Occupational Radiation Doses due to Intakes of Radionuclides. Safety Reports Series 37, 2004

[5] VIM - Vocabulário Internacional de Metrologia, Instituto Português de Qualidade. $1^{\text {a }}$ Ed. LusoBrasileira, 2012

[6] ICRP - International Commission on Radiological Protection. Individual Monitoring for Internal Exposure of Workers. Publication 78, 1998

[7] BERTELLI, L.; MELO, D.R.; LIPSZTEIN, J.; CRUZ-SUAREZ, R. AIDE: Internal Dosimetry Software" Radiation Protection Dosimetry v. 130 (3), p. 35, 2008 